\title{
Aqueous flare and cells in Fuchs syndrome
}

\begin{abstract}
Purpose To quantitatively evaluate aqueous flare and cells in patients with Fuchs syndrome. Methods The medical records of 40 patients (47 eyes) diagnosed with Fuchs syndrome between February 2006 and January 2007 at the Uveitis Study Center of Sun Yat-sen University were retrospectively reviewed. Aqueous flare and cells were clinically evaluated and quantified with laser flare-cell meter. Statistical analysis was performed to investigate the relationship between flare values and cell counts, and clinical parameters including patients' age, sex, duration of disease, best-corrected visual acuity, keratic precipitate, iris depigmentation, intraocular pressure, and posterior subcapsular lens opacities.
\end{abstract}

Results Aqueous flare values (photon counts/ ms) were significantly higher in Fuchs syndrome $(9.40 \pm 5.85)$ than in normal controls (5.77 $\pm 1.89, P=0.000)$. Aqueous cell counts (cells $/ 0.5 \mathrm{~mm}^{3}$ ) were also significantly higher in Fuchs syndrome $(5.09 \pm 4.84)$ than in normal controls $(1.14 \pm 1.03, P=0.000)$. The flare values were positively correlated with the cell counts ( $r=0.331, P=0.001)$. Both flare values and cell counts were higher in eyes with keratic precipitates scored $2+$ or $3+$ as compared to those with a $1+$ score. Higher flare values and cell counts were also observed in eyes with a $2+$ or $3+$ iris depigmentation score as compared to those with a $1+$ score. No difference was found between flare values and cell counts and other parameters.

Conclusion Breakdown of blood-aqueous barriers and increased cell counts are present in the affected eyes in patients with Fuchs syndrome. These changes are positively associated with the degree of keratic precipitates and iris depigmentation. Eye (2009) 23, 79-84; doi:10.1038/sj.eye.6702991; published online 26 October 2007

Keywords: laser flare-cell meter; Fuchs syndrome; uveitis

\author{
W Fang ${ }^{1,2,3,5}$, H Zhou ${ }^{1,2,3,5}$, P Yang ${ }^{1,2,3}$, X Huang ${ }^{1,2,3}$, \\ L Wang ${ }^{1,2,3}$ and A Kijlstra, ${ }^{2,4}$
}

Introduction

The term Fuchs syndrome, usually referred to Fuchs heterochromic iridocyclitis, is used for a specific uveitis entity usually manifesting as a mild unilateral anterior uveitis with stellate or medium-sized keratic precipitates (KP) and varying degrees of iris depigmentation or heterochromia. ${ }^{1-5}$ It occurs mostly in the third or fourth decades of adults and affects both sexes equally. Complicated cataract and secondary glaucoma are common in these patients. With careful slit-lamp examination, signs of intraocular inflammation including $\mathrm{KP}$, flare and cells in the anterior chamber, iris depigmentation or heterochromia are detectable in most affected eyes. Alteration of the bloodaqueous barrier (BAB) in patients with Fuchs syndrome has been demonstrated by iris fluorescein angiography. ${ }^{6}$ Our recent study using laser flare-cell meter (LFCM) analysis has confirmed that aqueous flare values and cell counts are increased in the affected eyes with Fuchs syndrome. ${ }^{7}$ However, it is not yet known which clinical parameters, such as patients' age, sex, laterality, best-corrected visual acuity (BCVA), duration of disease, $\mathrm{KP}$, iris depigmentation, intraocular pressure, and posterior subcapsular lens opacities are associated with these changes. In the present study, we quantitatively evaluated the breakdown of the BAB and the changes in the cells in the anterior chamber of the affected eyes in a consecutive series of patients with Fuchs syndrome and evaluated the relationship between the LFCM results and clinical parameters.

\section{Methods}

Forty patients with Fuchs syndrome referred to the Uveitis Study Center of the Sun Yat-sen University were included in this study. The diagnosis of Fuchs syndrome was based on clinical biomicroscopic findings including chronic anterior uveitis with low inflammatory

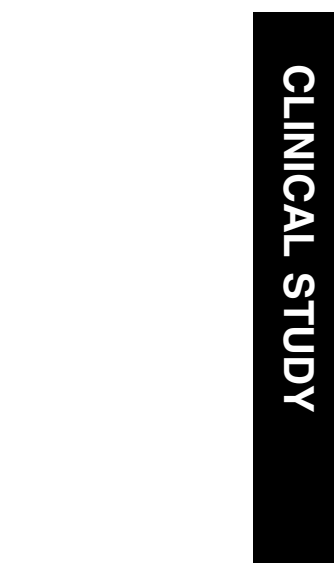

'Zhongshan Ophthalmic Center, Sun Yat-sen University, Guangzhou, P.R. China

${ }^{2}$ Uveitis Study Center and International Uveitis Study Laboratory of Sun Yat-sen University, Guangzhou, P.R. China

${ }^{3}$ State Key Laboratory of Ophthalmology of Sun Yat-sen University, Guangzhou, P.R. China

${ }^{4}$ Department of Ophthalmology, Eye Research Institute Maastricht, University Hospital Maastricht,

Maastricht, The Netherlands

Correspondence: P Yang, Zhongshan Ophthalmic Center,

Eye Research Institute, 54 South Xianlie Road Guangzhou 510060,

P.R. China

Tel: + 00862087330402 ; Fax: + 00862087330403 .

E-mail: peizengyang@ hotmail.com

${ }^{5}$ Both these authors contributed equally to the study.

Received: 8 March 2007 Accepted in revised form: 2 September 2007

Published online: 26 October 2007

None of the authors has a proprietary or financial interest in any product mentioned. 
activity, characteristic stellate or medium-sized KP diffusely or centrally distributed on the corneal endothelium, diffuse iris depigmentation or heterochromia, and lack of posterior synechiae..$^{7-9}$ Idiopathic chronic anterior uveitis, Posner-Schlossman syndrome, and other conditions that may cause impairment of the BAB (diabetes mellitus, pseudoexfoliation, occlusion of retinal vessels, ocular trauma, intraocular tumors, and so on) were excluded from this study through careful examination. In cases of diagnostic doubt, we performed HLA-B27 typing in suspected patients to rule out HLA-B27-associated anterior uveitis. Serum angiotensin-converting enzyme assay and chest X-ray were performed in suspected patients to rule out sarcoidosis.

A detailed history, including age, laterality, complaints, the BCVA, and associated systemic diseases, was taken from each patient. All patients were examined by the same uveitis specialist. The examinations included the BCVA, slit-lamp biomicroscopy for bilateral anterior segment analysis of the eye, gonioscopy, and

ophthalmoscopy through a dilated pupil. The severity of anterior chamber activity was clinically evaluated using the grading system proposed by the Standardization of Uveitis Nomenclature (SUN) Working Group. ${ }^{10}$ The degree of $\mathrm{KP}$ was defined as $(0,1+, 2+, 3+$, and $4+)$ according to the number of $\mathrm{KP}$, as described by La Hey et $a l^{9}$ and Hogan et $a l^{11}$. Iris depigmentation was identified as $0,1+, 2+$, and $3+$ based on the colour and depigmentation of the iris (Table 1; Figures 1 and 2). Intraocular pressure was measured using the noncontact tonometry method. Most patients received nonsteroid anti-inflammatory eye drops topically when anterior chamber reaction was present. Few patients received corticosteroid eye drops for a short time because of the presence of overt anterior chamber cells $(1+)$. None of the tested patients received topical or systemic steroids treatment at least for 5 days before examination by LFCM.

Table 1 Degree of iris depigmentation under slit-lamp biomicroscopy in the present study

\begin{tabular}{ll}
\hline Grade & Description \\
\hline 0 & $\begin{array}{l}\text { No depigmentation } \\
\text { Depigmentation is visible only when comparing the } \\
\text { iris of both eyes. Mild transillumination around the } \\
\text { light beam on the iris is present } \\
\text { Depigmentation is visible at glance under slit-lamp } \\
\text { biomicroscopy. Transillumination is moderate around } \\
\text { the light beam on the iris } \\
\text { Depigmentation is marked and, subtle heterochromia } \\
\text { could be clearly observed, transillumination is } \\
\text { obvious }\end{array}$ \\
\end{tabular}

The quantitative measurement of aqueous flare and cells was performed by an experienced technician who did not know the clinical results. All data were acquired using standardized data sheets that were designed before the study was started. LFCM was performed on 47 eyes
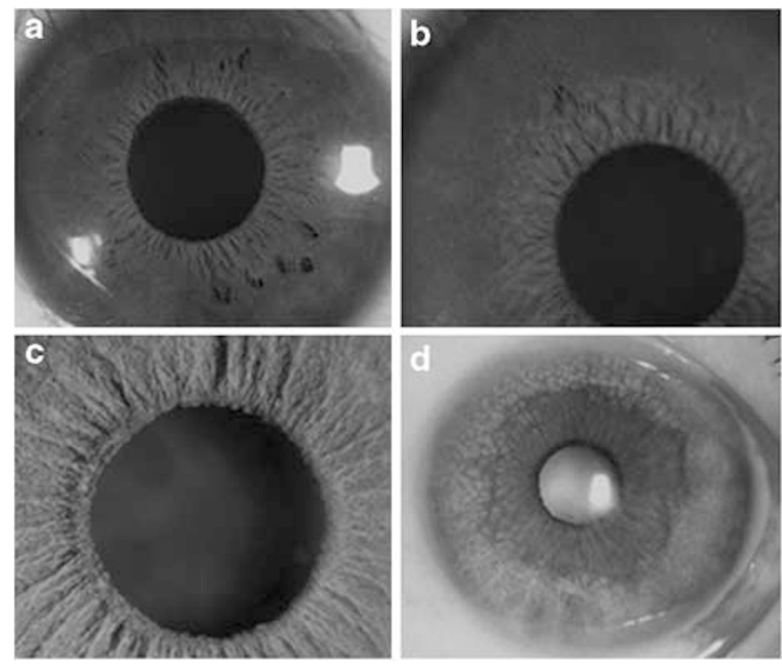

Figure 1 Slit-lamp photography with diffuse illumination showing various degrees of iris depigmentation in normal controls and patients with Fuchs syndrome: (a) Normal iris appearance without depigmentation. (b) Iris depigmentation $(1+)$. (c) Iris depigmentation $(2+)$. (d) Iris depigmentation $(3+)$ with 'moth-eaten' appearance.
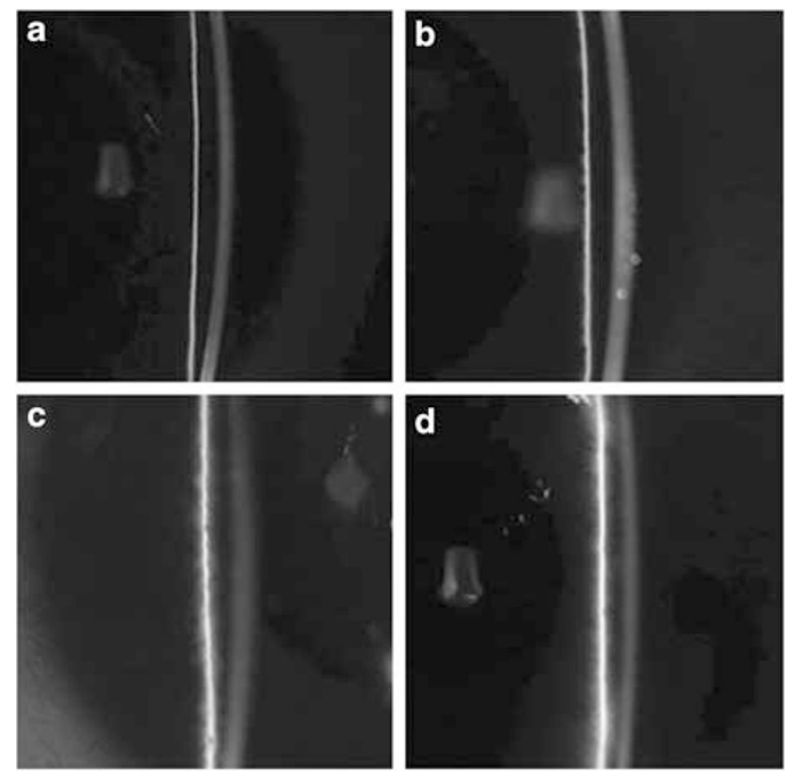

Figure 2 Slit-lamp photography with narrow slit showed various degrees of transillumination in normal controls and patients with Fuchs syndrome: (a) Normal iris appearance without transillumination. (b) Mild transillumination with iris depigmentation $(1+)$. (c) Moderate transillumination with iris depigmentation $(2+)$. (d) Obvious transillumination with iris depigmentation $(3+)$. 
of 40 cases using the FC-2000 LFCM (version 1.0) (Kowa Company Ltd, Tokyo, Japan) to quantitatively evaluate aqueous flare and cells. This examination was also concurrently performed on 47 eyes of 36 normal individuals as age-matched controls. Three individual measurements were averaged and those with artifacts were discarded. The results of flare values and cell counts were expressed as photon counts/ms (pc/ms) and cells $/ 0.5 \mathrm{~mm}^{3}$, respectively. The results were given as mean \pm SD and were analysed using the software of SPSS 11.0. The statistical methods used in this study included the $t$-test, nonparametric correlation analysis (Bivariate Kendall method), and one-way ANOVA analysis (Post hoc multiple comparisons).

We certify that all applicable institutional and governmental regulations concerning the ethical use of human volunteers were followed during this research. The study and data accumulation were carried out with approval from the Institutional Review Board (IRB) of Zhongshan Ophthalmic Center. All patients and healthy volunteers were informed about the nature of the noninvasive examinations of LFCM and consented to this examination.

\section{Results}

A total of 47 eyes with Fuchs syndrome from 40 patients (15 males, 25 females, mean age $33.8 \pm 10.1$ years, range 16-72 years) and 47 normal control eyes of 36 healthy individuals (13 males, 23 females, mean age $34.8 \pm 12.1$ years, range 17-66 years) were enrolled in this study. The duration of disease (mainly according to the patients' complaints) varied from 17 days to 20 years, with a median of 3 years. The BCVA of Fuchs eyes ranged from $0.1(20 / 200)$ to $1.5(20 / 13.3)$, with a mean BCVA of $0.87 \pm 0.44(20 / 23 \pm 20 / 45)$.

Clinically, the anterior chamber reaction was mild in these patients with Fuchs syndrome. Flare $(+)$ was observed in $72.3 \%$ (34 of 47) of affected eyes. More than half of eyes $(53.2 \%, 25$ of 47$)$ had no detectable cells in the anterior chamber. Aqueous cells $(1+)$ and $(0.5+)$ were observed in $29.8 \%$ (14 of 47 ) and $17.0 \%$ (8 of 47 ) of eyes. There were $7,17,14$, and 9 eyes graded as KP $(0+)$, $(1+),(2+)$, and $(3+)$, respectively. Among the eyes with $\mathrm{KP}$, more than half showed a diffuse distribution (65\%) and stellate morphology (52.5). Iris depigmentation was graded as $(1+),(2+)$, and $(3+)$ in 19, 20 and, 8 eyes, respectively. Posterior subcapsular lens opacity was present in less than $30 \%$ of eyes.

\section{LFCM flare values and cell counts in patients and controls}

The mean flare values in 40 patients and 36 controls were $9.40 \pm 5.85$ and $5.77 \pm 1.89 \mathrm{pc} / \mathrm{ms}$. The number of anterior chamber cells in 40 patients and 36 controls was $5.09 \pm 4.84$ cells $/ 0.5 \mathrm{~mm}^{3}$ and $1.14 \pm 1.03$ cells $/ 0.5 \mathrm{~mm}^{3}$, respectively. There was a significant difference between patients and controls with regard to flare values ( $t=4.041, P=0.000)$ and cell counts $(t=6.465, P=0.000)$ in the anterior chamber.

\section{LFCM flare values vs cell counts}

The LFCM results showed a good association between flare values and cell counts. There was a significant correlation between these two parameters (Kendall's correlation coefficient, $r=0.331, P=0.001$; Figure 3).

\section{LFCM flare values and cell counts vs slit-lamp gradings}

Figure 4 shows the relation between the LFCM results and slit-lamp gradings of anterior inflammation in patients with Fuchs syndrome. Flare values ranged from 1.9 to $34.9 \mathrm{pc} / \mathrm{ms}$ and slit-lamp gradings varied from $(0+)$ to $(1+)$. There was a good correlation between the LFCM results and slit-lamp readings in flare measurements (Kendall's correlation coefficient, $r=0.402, P=0.001)$. Higher cell counts were also noted in the eyes with higher gradings by slit-lamp analysis (Kendall's correlation coefficient, $r=0.756, P=0.000$ ).

\section{LFCM flare values and cell counts vs degree of KP}

LFCM flare values and cell counts $v$ degree of KP in Fuchs syndrome are shown in Table 2. There was a good correlation between the results of flare values and slitlamp gradings of KP (Kendall's correlation coefficient, $r=0.381, P=0.001)$. There was also a good correlation

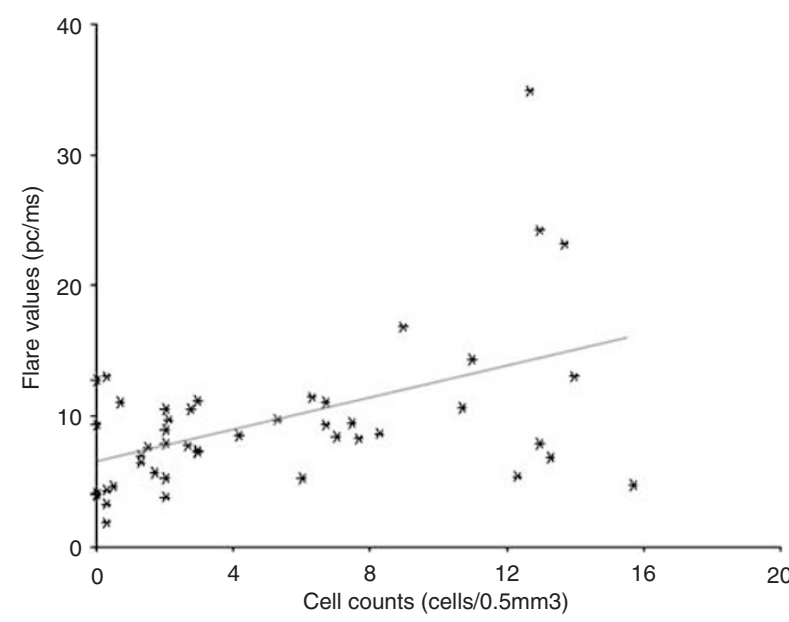

Figure 3 Correlation between flare values and cell counts in patients with Fuchs syndrome (Kendall's correlation coefficient: $r=0.331, P=0.001$ ). 

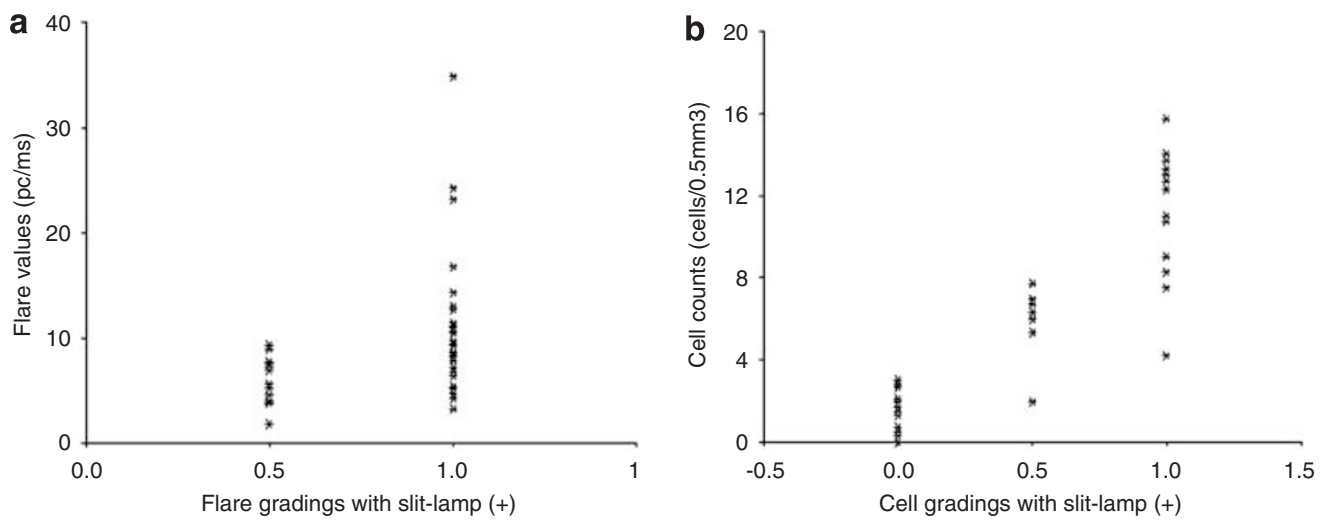

Figure 4 Correlation between flare values (a) (Kendall's correlation coefficient: $r=0.402, P=0.001$ ) and cell counts (b) (Kendall's correlation coefficient: $r=0.756, P=0.000)$ vs slit-lamp gradings in patients with Fuchs syndrome.

Table 2 The results of LFCM and degree of KP in Fuchs patients

\begin{tabular}{lrcr}
\hline $\begin{array}{l}\text { Degree } \\
\text { of } K P\end{array}$ & Eyes & $\begin{array}{c}\text { Flare values } \\
(p c / m s)\end{array}$ & \multicolumn{1}{c}{$\begin{array}{c}\text { Cell counts } \\
\left(\text { cells } / 0.5 \mathrm{~mm}^{3}\right)\end{array}$} \\
\hline $0+$ & 7 & $5.44 \pm 2.71$ & $1.27 \pm 1.16$ \\
$1+$ & 17 & $7.61 \pm 2.85$ & $2.28 \pm 1.96$ \\
$2+$ & 14 & $11.23 \pm 4.76$ & $6.38 \pm 4.05$ \\
$3+$ & 9 & $13.00 \pm 9.87$ & $12.02 \pm 2.70$ \\
\hline
\end{tabular}

Abbreviations: LFCM, laser flare-cell meter; KP; keratic precipitates.

between the results of LFCM cell counts and slit-lamp gradings of KP (Kendall's correlation coefficient, $r=0.696, P=0.000)$. These results suggest that increased flare values and cell counts are present in eyes with higher slit-lamp gradings of KP.

\section{LFCM flare values and cell counts vs degree of iris depigmentation}

Flare values and cell counts vs degree of iris depigmentation in Fuchs syndrome are shown in Table 3. There was a good correlation between the results of flare values and slit-lamp gradings of iris depigmentation (Kendall's correlation coefficient, $r=0.441, P=0.000$ ). A similar result was also observed between cell counts and slit-lamp gradings of iris depigmentation (Kendall's correlation coefficient, $r=0.482, P=0.000$ ).

\section{LFCM flare values and cell counts vs other clinical parameters}

With respect to the distribution of $\mathrm{KP}$, we noted significantly higher flare values and cell counts in eyes with diffusely distributed KP compared to those with centrally distributed KP (13.26 vs $6.45 \mathrm{pc} / \mathrm{ms}, P=0.048$; 7.08 vs 2.50 cells $\left./ 0.5 \mathrm{~mm}^{3}, P=0.037\right)$. However, there
Table 3 The results of LFCM and iris depigmentation in Fuchs patients

\begin{tabular}{lrrr}
\hline $\begin{array}{l}\text { Degree of iris } \\
\text { depigmentation }\end{array}$ & Eyes & \multicolumn{1}{c}{$\begin{array}{c}\text { Flare values } \\
(p c / m s)\end{array}$} & \multicolumn{1}{c}{$\begin{array}{c}\text { Cell counts } \\
\left(\text { cells/0.5 } \mathrm{mm}^{3}\right)\end{array}$} \\
\hline $0+$ & 33 & $5.19 \pm 2.14$ & $0.86 \pm 0.63$ \\
$1+$ & 19 & $6.30 \pm 2.76$ & $1.96 \pm 2.21$ \\
$2+$ & 20 & $10.36 \pm 4.24$ & $5.99 \pm 4.64$ \\
$3+$ & 8 & $14.36 \pm 9.99$ & $10.26 \pm 4.86$ \\
\hline
\end{tabular}

Abbreviation: LFCM, laser flare-cell meter.

were no differences either between patients with diffusely distributed KP and those with inferiorly distributed KP or between patients with centrally distributed KP and those with inferiorly distributed KP concerning flare values and cell counts.

No differences were observed between flare values, cell counts, and other parameters, including patients' age, sex, laterality, BCVA, duration of disease, morphology of $\mathrm{KP}$, intraocular pressure, and posterior subcapsular lens opacities.

\section{Discussion}

In this study, we used LFCM to quantitatively evaluate the breakdown of the $\mathrm{BAB}$ and cell reaction in the anterior chamber in patients with Fuchs syndrome. Our study showed that patients with Fuchs syndrome display significantly higher flare values and cell counts than normal controls. Further analysis showed that these changes were positively associated with the degree of KP and iris depigmentation. However, these changes were not linked with other clinical parameters, such as patients' age, sex, laterality, BCVA, duration of disease, morphology of $\mathrm{KP}$, intraocular pressure, or posterior subcapsular lens opacities. 
The Kowa LFCM has been proven to be a reliable tool in quantifying aqueous flare and cells in vivo by measuring light scattering from a low-power He-Ne beam. The accuracy and reproducibility of the method have been shown in several studies. ${ }^{12-23}$ Using LFCM, we recently showed breakdown of the $\mathrm{BAB}$ and presence of anterior chamber cells in Chinese patients with Fuchs syndrome. ${ }^{7}$ The present study confirmed our previous observation in another group of Chinese patients with Fuchs syndrome using the same technique. These results suggest that breakdown of the BAB and increased cell counts are a prominent feature of this disease.

In the present study, the LFCM results showed a good correlation with a clinical grading system. These results, on the one hand, indicate that careful slit-lamp examination provides a simple and rapid assessment for the anterior reaction. On the other hand, the LFCM may provide quantitative information for the evaluation of the anterior inflammation. Our results also showed that the flare values were positively associated with the cell counts. These results show that breakdown of BAB in this disease can directly be attributed to the degree of inflammation in the anterior chamber.

To clarify which clinical parameters could influence the results of LFCM, we divided patients into subgroups according to a number of factors including patients' age, sex, duration of disease, BCVA, KP, iris depigmentation, intraocular pressure, and posterior subcapsular lens opacities. Our results showed that the flare values and cell counts were associated with the degree of KP. As the presence of $\mathrm{KP}$ is one of the important indexes of inflammation in the anterior chamber, it is not difficult to understand the positive link between them. We further analysed the association between morphology and distribution of KP and LFCM results. Our results showed no relationship between the morphology of KP and the LFCM results. Interestingly, we found a strong association of LFCM results with diffusely distributed $\mathrm{KP}$. This result may be explained by the fact that the diffusely distributed KP are usually seen in eyes with KP score $2+$ and $3+$ (19 of 26 eyes).

Another parameter that was associated with flare values and cell counts was the degree of iris depigmentation. Since iris depigmentation has been considered as a sequence of long-lasting or chronic anterior inflammation in Fuchs syndrome, it is obvious that this sign may represent, to a certain degree, the activity of the anterior chamber reaction. These findings support the theory that the chronic anterior chamber inflammation is responsible for the iris depigmentation. ${ }^{24}$

It is interesting to note that the flare values and cell counts were not associated with other clinical parameters, for instance, patients' age, sex, laterality, BCVA, intraocular pressure, and posterior subcapsular lens opacities. Unexpectedly, the breakdown of BAB indirectly revealed by LFCM was not found to be associated with the disease duration. This result could be misinterpreted by the uncertainty of the disease duration described by patients themselves. More importantly, this result indicates that the breakdown of $\mathrm{BAB}$ in these patients is mainly caused by the active anterior inflammation. Interestingly, our results show that the LFCM readings are not associated with the presence of posterior subcapsular lens opacity. We suppose this relationship would have existed if chronic inflammation were a sole factor contributing to posterior subcapsular lens opacity. Actually we failed to find this relationship in this study. Therefore, other factors, such as prolonged use of topical steroids eye drops before referring to us, might also contribute to the development of posterior subcapsular lens opacity.

In conclusion, our study characterized the anterior chamber reaction in patients with Fuchs syndrome using LFCM. The results showed a concurrent presence of elevated flare values and cell counts. There was a strong association between LFCM results and the degree of KP and iris depigmentation, both mostly indicative of the inflammation in the anterior chamber. The combined results suggest that the low-grade inflammation in the anterior chamber is responsible for the BAB breakdown observed in patients with Fuchs syndrome.

\section{Acknowledgements}

This study was supported in part by the Fund for Project of Science and Technology of Guangdong province (2005B60302009), Key Project of Natural Science Foundation (30630064), National supporting project of P.R. China, and '5010' Clinical Project of Sun Yat-sen University.

\section{References}

1 Loewenfeld IE, Thompson S. Fuchs heterochromic cyclitis: a critical review of the literature. I. Clinical characteristics of the syndrome. Surv Ophthalmol 1973; 17: 394-457.

2 Jones NP. Fuchs heterochromic uveitis: a reappraisal of the clinical spectrum. Eye 1991; 5: 649-661.

3 Jones NP. Fuchs heterochromic cyclitis: an update. Surv Ophthalmol 1993; 37: 253-272.

4 Fearnley IR, Rosenthal AR. Fuchs heterochromic iridocyclitis revisited. Acta Ophthalmol Scand 1995; 73: 166170.

5 Mohamed Q, Zamir E. Update on Fuchs' uveitis syndrome. Curr Opin Ophthalmol 2005; 16: 356-363.

6 Norrsell K, Holmer AK, Jacobson H. Aqueous flare in patients with monocular iris atrophy and uveitis. A laser flare and iris angiography study. Acta Ophthalmol Scand 1998; 76: 405-412. 
7 Yang P, Fang W, Jin H, Li B, Chen X, Kijlstra A. Clinical features of Chinese patients with Fuchs syndrome. Ophthalmology 2006; 113: 473-480.

8 Kimura SJ, Hogan MJ, Thygeson P. Fuchs syndrome of heterochromic cyclitis. Arch Ophthalmol 1955; 54: 179-186.

9 La Hey E, Baarsma GS, De Vries J, Kijlstra A. Clinical analysis of Fuchs heterochromic cyclitis. Doc Ophthalmol 1991; 78: 225-235.

10 The Standardization of Uveitis Nomenclature (SUN) Working Group. Standardization of uveitis nomenclature for reporting clinical data. Am J Ophthalmol 2005; 118: 338-342.

11 Hogan MJ, Kimura SJ, Thygeson P. Signs and symptoms of uvietis. I Anterior uveitis. II classification of the posterior manifestations of uveitis. Am J Ophthalmol 1959; 47: 155-176.

12 Ohara K, Okubo A, Miyazawa A, Miyamoto T, Sasaki H, Oshima F. Aqueous flare and cell measurement using laser in endogenous uveitis patients. Jpn J Ophthalmol 1989; 33: 265-270.

13 Oshika T, Nishi M, Mochizuki M, Nakamura M, Kawashima H, Iwase $\mathrm{K}$ et al. Quantitative assessment of aqueous flare and cells in uveitis. Jpn J Ophthalmol 1989; 33: 279-287.

14 Yoshitomi T, Wong SA, Daher E, Sears LM. Aqueous flare measurement with laser flare-cell meter. Jpn J Ophthalmol 1990; 34: 57-62.

15 Sawa M. Clinical application of laser flare-cell meter. Jpn J Ophthalmol 1990; 34: 346-363.

16 Guex-Crosier Y, Pittet N, Herbort PC. Evaluation of laser flare-cell photometry in the appraisal and management of intraocular inflammation in uveitis. Ophthalmology 1994; 101: 728-735.

17 Guex-Crosier Y, Pittet N, Herbort PC. Sensitivity of laser flare photometry to monitor inflammation in uveitis of the posterior segment. Ophthalmology 1995; 102: 613-621.

18 Saari MK, Guillen-Monterrubio MO, Hartikainen J, Hamalainen MM, Taskinen K. Measurement of protein concentration of aqueous humour in vivo: correlation between laser flare measurements and chemical protein determination. Acta Ophthalmol Scand 1997; 75: 63-66.

19 Kuchle M, Nguyen NX, Horn F, Naumann GOH. Quantitative assessment of aqueous flare and aqueous "cells" in pseudo-exfoliation syndrome. Acta Ophthalmol 1992; 70: 201-208.

20 Kuchle M, Nguyen NX, Naumann GOH. Quantitative assessment of the blood-aqueous barrier in human eyes with malignant or benign uveal tumors. Am J Ophthalmol 1994; 117: 521-528.

21 Kuchle M, Nguyen NX, Martus P, Freissler K, Schalnus R. Aqueous flare in retinitis pigmentosa. Graefe's Arch Clin Exp Ophthalmol 1998; 236: 426-433.

22 Jandrasits K, Krepler K, Wedrich A. Aqueous flare and macular edema in eyes with diabetic retinopathy. Ophthalmologica 2003; 217: 49-52.

23 Nguyen NX, Kuchle M, Naumann GOH. Quantification of blood-aqueous barrier breakdown after phacoemulsification in Fuchs' heterochromic uveitis. Ophthalmologica 2005; 219: 21-25.

24 Bonfioli AA, Curi LLA, Orefice F. Fuchs' heterochromic cyclitis Semin. Ophthalmol 2005; 20: 143-146. 